\section{Revistas científicas de estudiantes de medicina en Latinoamérica}

\author{
IGNACIO CABRERA-SAMITH ${ }^{1}$, DIANA ORÓSTEGUI-PINILLA ${ }^{2}$, \\ YOLANDA ÁNGULO-BAZÁN ${ }^{3}$, PERCY MAYTA-TRISTÁN ${ }^{4}$, \\ ALFONSO J. RODRÍGUEZ-MORALES ${ }^{5}$, en nombre del grupo \\ de Editores de revistas científicas estudiantiles de Latinoamérica.
}

\section{Scientific journals of medical students in Latin-America}

This article deals with the history and evolution of student's scientific journals in Latin-America, their beginnings, how many still exist and which is their future projection. Relevant events show the growth of student's scientific journals in LatinAmerica and how are they working together to improve their quality. This article is addressed not only for Latin American readers but also to worldwide readers. Latin American medical students are consistently working together to publish scientific research, whose quality is constantly improving.

(Rev Med Chile 2010; 138: 1451-1455).

Key words: Periodicals as topic; Research.

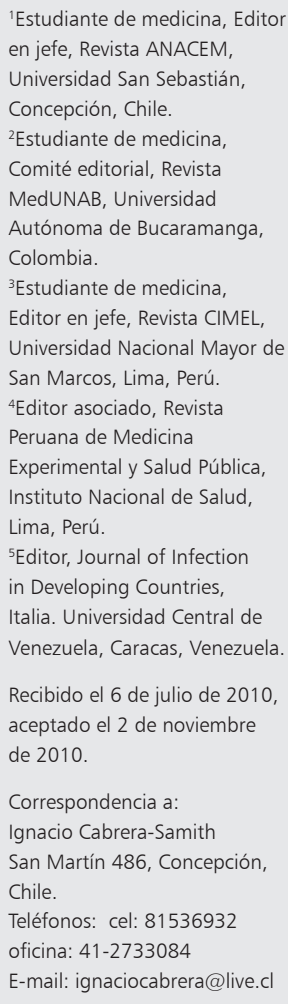

$\mathrm{D}$ urante la formación de los estudiantes de medicina el desarrollo de sus habilidades científicas es tan importante como el de sus habilidades clínicas, ya que el conocimiento sobre el método científico y la investigación en salud puede ser muy útil en la atención médica. Por lo tanto, la investigación y publicación científica es un punto muy relevante en la formación de un estudiante de medicina ${ }^{1,2}$. Las escuelas médicas modernas incorporan tanto el método científico como la práctica clínica en su educación básica clínica/científica. Esto es muy importante para la formación de futuros médicos que puedan practicar la Medicina Basada en la Evidencia (MBE). El futuro de la MBE no solamente se basa en el conocimiento del método científico sino también incluye una participación activa de los médicos en la investigación. La investigación extracurricular ha sido largamente impulsada en las escuelas de medicina y ha sido reconocida como un determinante importante en la decisión de continuar posteriormente con la investigación en el postgrado ${ }^{3,4}$. Además, la investigación extracurricular ayuda en el mejoramiento de las habilidades científicas en los estudiantes de pregrado y postgrado. Sin embargo, la producción científica puede ser potenciada por diferentes estímulos. En este sentido, desde un punto de vista pragmático, podemos decir que para un estudiante de medicina es muy gratificante publicar un manuscrito. Desafortunadamente esto no es un proceso fácil, ya que son muchos los problemas y obstáculos que se deben superar para alcanzar esta meta, que pueden demorar el proceso de investigación realizado por los estudiantes de medicina. Estos incluyen (no exclusivamente): falta de una buena asesoría docente, falta de estudios rutinarios, falta de tiempo por la sobrecarga académica, deterioro en la habilidades científicas debido a mayor tiempo ocupado en actividades clínicas y manejo inadecuado de la metodología de proyectos de investigación. Otro problema frecuente que siempre es discutido por aquellos que critican la investigación estudiantil de pregrado es que los manuscritos que son publicados por los estudiantes son raramente citados en revistas importantes, limitando su utilidad e impacto ${ }^{5-7}$. 
En respuesta a estos problemas, grupos de estudiantes de medicina en Latinoamérica, se han reunido para crear sus propias revistas científicas y han trabajado para mejorar la calidad de sus investigaciones. Las revistas científicas estudiantiles han sido largamente reconocidas como una oportunidad para los estudiantes de pregrado para expresar sus ideas de investigación. A pesar de esto, el impacto de las revistas científicas estudiantiles como estímulo del interés en la investigación biomédica no ha sido siempre comprendido ni adecuadamente asesorado. Las revistas estudiantiles se presentan como una oportunidad única para comprometer tempranamente a los estudiantes con un nivel más personal y comprensivo en la investigación. Dado el hecho que han sido creadas especialmente para conocer los requerimientos de los estudiantes de medicina y facilitarles el proceso de investigación y publicación científica, es reconocido por muchos estudiantes que el proceso de publicar un artículo científico es difícil. Las políticas de publicación de las revistas científicas estudiantiles prestan especial atención a la publicación de artículos por estudiantes que están embarcados en su carrera de investigación, acogiéndolos, orientándolos y estimulándolos. Incluso si no poseen acceso a recolectar datos científicos para confeccionar un artículo original de investigación, se les da la oportunidad de realizar otro tipo de artículos para contribuir al conocimiento biomédico, tales como revisiones de temas, cartas al editor, reporte de casos, entre otros, siempre bajo la asesoría de un buen docente. Las cartas de apreciación crítica pueden, frecuentemente, demostrar la competencia de los estudiantes en el uso del método científico, estimulando su interés en la vertiente académica y científica de la medicina. Muchos estudiantes, incluyendo los de Latinoamérica, han publicado sus primeros artículos en revistas estudiantiles e incluso han logrado durante sus estudios de pregrado publicar algún artículo en revistas científicas de alto impacto ${ }^{8}$.

\section{¿Qué está sucediendo en Latinoamérica?}

Es importante discutir el estado de las revistas científicas estudiantiles en la región de Latinoamérica. Esta es una área geográfica que comparte muchos aspectos educacionales y socioculturales con la mayoría de los países considerados en desarrollo, llegando a trabajar en interesantes iniciativas que se han generado en los últimos veinte años, promoviendo la investigación y publicación científica.

A diferencia de otras regiones del mundo, donde la mayoría de las publicaciones en las revistas estudiantiles son comentarios o revisiones, en Latinoamérica la gran parte de las revistas científicas estudiantiles están enfocadas a publicar artículos de investigación hecha por estudiantes de medicina $^{8,9}$. La corriente de trabajo de las revistas científicas estudiantiles en Latinoamérica debe ser considerada, hoy en día, muy potente, en contraste con otras regiones del mundo donde se publican artículos en formato de opiniones, puntos de vista y hechos médicos escritos por estudiantes de medicina. También transmiten las actividades de ciertas instituciones estudiantiles, pero no se publican manuscritos originales de investigación. Entre estas revistas podemos mencionar el British Medical Journal Student (www.studentbmj.com) del Reino Unido, Vagus, la Revista de la Federación Internacional de Asociaciones de Estudiantes de Medicina (IFMSA) (www.ifmsa.org/publications), la española "Protomédicos" (www.protomedicos. com) y la Trinity Student Medical Journal (www. tcd.ie/tsmj) de Irlanda.

En Latinoamérica, los estudiantes de medicina han trabajado en conjunto para mejorar su producción científica, particularmente a través de promover la presentación de trabajos de investigación en congresos científicos, desde 1986, cuando se creó la Federación Latinoamérica de Sociedades Científicas de Estudiantes de Medicina (FELSOCEM). El principal evento de FELSOCEM es el Congreso Científico Internacional (CCI), donde los estudiantes latinoamericanos de diferentes ciudades y países se reúnen anualmente para presentar los resultados de sus investigaciones y su producción científica, siendo apoyada no solamente por sus universidades sino también por organizaciones locales, regionales o nacionales llamadas Sociedades Científicas de Estudiantes de Medicina (SOCEM).

De este modo, siendo la publicación científica la etapa final y más importante del proceso de investigación científica es que durante el año 1998, en el seno del CCI de FELSOCEM se creó un nuevo evento, llamado Encuentro Internacional de Revistas Científicas Estudiantiles de Ciencias de la Salud (EIRCECS), para discutir múltiples aspectos relacionados con la metodología del proceso editorial, de la revisión por pares, de la publicación y discusión de temas sobre ética, de la creación de 
Revistas científicas de estudiantes de medicina - I. Cabrera-Samith et al

redes de trabajos para editores y la promoción de la importancia de trabajar para mejorar la calidad de las publicaciones ${ }^{10}$. La Tabla 1 resume los principales aspectos relacionados con el desarrollo de estos encuentros.
En nuestra región, se han creado al menos 20 revistas científicas de estudiantes de medicina, la mayoría de ellas persisten activas en la actualidad, representando al menos nueve países, con una revista internacional regional llamada Ciencia e

Tabla 1. Evolución de los Encuentros Internacionales de Revistas Científicas de Ciencias de la Salud (EIRCECS) $^{10}$

\begin{tabular}{|c|c|c|c|}
\hline Año & Encuentro & Lugar & Resumen \\
\hline 1998 & I & $\begin{array}{l}\text { Cartagena, } \\
\text { Colombia }\end{array}$ & $\begin{array}{l}\text { Reunión amical entre tres editores de revistas estudiantiles, durante el CCI. Se } \\
\text { enteraron de la existencia de otras revistas estudiantiles y acordaron organizar un } \\
\text { encuentro el próximo año }\end{array}$ \\
\hline 1999 & II & $\begin{array}{l}\text { Panamá, } \\
\text { Panamá }\end{array}$ & $\begin{array}{l}\text { La Revista Médico Científica organiza el primer encuentro formal dentro del CCI. } \\
\text { La asistencia fue baja, pero los editores lograron discutir temas sobre la publica- } \\
\text { ción de artículos científicos }\end{array}$ \\
\hline 2000 & III & $\begin{array}{l}\text { Asunción, } \\
\text { Paraguay }\end{array}$ & $\begin{array}{l}\text { Se acepta la inclusión permanente de los EIRCECS en el marco de los CCI. David } \\
\text { Sharp, editor de la Revista LANCET, participa como expositor. Durante este en- } \\
\text { cuentro se funda la revista Medicina Actual de Paraguay y se firma un acuerdo } \\
\text { llamado la Declaración de Paraguay }\end{array}$ \\
\hline 2001 & IV & $\begin{array}{l}\text { Mérida, } \\
\text { Mexico }\end{array}$ & $\begin{array}{l}\text { Durante este año no hubo un encuentro formal. Los editores se reunieron infor- } \\
\text { malmente para compartir e intercambiar información }\end{array}$ \\
\hline 2002 & V & $\begin{array}{l}\text { Lima, } \\
\text { Perú }\end{array}$ & $\begin{array}{l}\text { Este ha sido el encuentro más grande, once revistas participaron activamente. Se } \\
\text { dio importancia a la publicación en el pregrado y a la revisión por pares, sirviendo } \\
\text { como base para crear el Comité Editorial de la Revista Ciencia e Investigación } \\
\text { Médica Estudiantil Latinoamericana, CIMEL }\end{array}$ \\
\hline 2003 & $\mathrm{VI}$ & $\begin{array}{l}\text { La Paz, } \\
\text { Bolivia }\end{array}$ & $\begin{array}{l}\text { Se realizaron plenarias sobre la redacción científica. Los editores discutieron so- } \\
\text { bre la importancia de trabajar en revistas nacionales, evitando crear más de una } \\
\text { revista por escuela de medicina. No hubo un acuerdo formal a esta propuesta }\end{array}$ \\
\hline 2004 & $\mathrm{VI}$ & $\begin{array}{l}\text { Antofagasta, } \\
\text { Chile }\end{array}$ & $\begin{array}{l}\text { Se realizó un taller sobre el proceso editorial. Los editores compartieron informa- } \\
\text { ción sobre los diferentes estilos de trabajo usados en la región }\end{array}$ \\
\hline 2005 & VIII & $\begin{array}{l}\text { Panamá, } \\
\text { Panamá }\end{array}$ & $\begin{array}{l}\text { Se contó con la participación de Plos Medicine, dándose énfasis en la importancia } \\
\text { del Open Access, la divulgación electrónica y la revisión por pares. Se realizó un } \\
\text { taller sobre el significado de las revistas estudiantiles. Se acordó que todas las } \\
\text { revistas deberían tener ISSN }\end{array}$ \\
\hline 2006 & IX & $\begin{array}{l}\text { Asunción, } \\
\text { Paraguay }\end{array}$ & $\begin{array}{l}\text { Se realizaron talleres de trabajo sobre las formas de revisión por pares y capaci- } \\
\text { tación de los comités editoriales }\end{array}$ \\
\hline 2007 & $x$ & $\begin{array}{l}\text { Mazatlán, } \\
\text { Mexico }\end{array}$ & $\begin{array}{l}\text { Este encuentro se concentró en la importancia del proceso editorial y la indexa- } \\
\text { ción nacional e internacional. Nuevamente se propuso trabajar solamente en } \\
\text { una revista a nivel nacional, evitando la creación de varias revistas por escuela de } \\
\text { medicina, sin llegar a acuerdo alguno }\end{array}$ \\
\hline 2008 & $\mathrm{XI}$ & $\begin{array}{l}\text { Iquique, } \\
\text { Chile }\end{array}$ & $\begin{array}{l}\text { Ha sido el encuentro más productivo de todos. Se realizaron talleres sobre el } \\
\text { proceso editorial y la indexación. Se contó con la participación como expositor } \\
\text { del Editor Jefe de la Revista Médica de Chile. Los editores discutieron sobre la } \\
\text { importancia de crear un grupo bien consolidado que reúna a todas las revistas } \\
\text { científicas estudiantiles de Latinoamérica. Sus acuerdos fueron: Creación de un } \\
\text { grupo de trabajo en línea activo entre editores. La indexación de todas la revistas } \\
\text { en IMBIOMED, SclELO y en el catálogo de Latindex. La futura creación de un } \\
\text { SciELO de estudiantes de pregrado, entre otras }\end{array}$ \\
\hline 2009 & XII & $\begin{array}{l}\text { La Paz, } \\
\text { Bolivia }\end{array}$ & $\begin{array}{l}\text { El principal tema de este encuentro fue el proceso editorial, realizándose un } \\
\text { pequeño taller sobre éste. Se revisaron los acuerdos cumplidos del año anterior } \\
\text { como la creación del grupo de trabajo en línea de editores y la publicación de un } \\
\text { artículo sobre los EIRCECS en la Revista Panamericana de Salud Pública. Se acor- } \\
\text { daron nuevos desafíos para el próximo año: creación de un SciELO para revistas } \\
\text { científicas estudiantiles y la organización de un taller sobre el proceso editorial en } \\
\text { el EIRCECS } 2010 \text { de Valparaíso, Chile }\end{array}$ \\
\hline
\end{tabular}




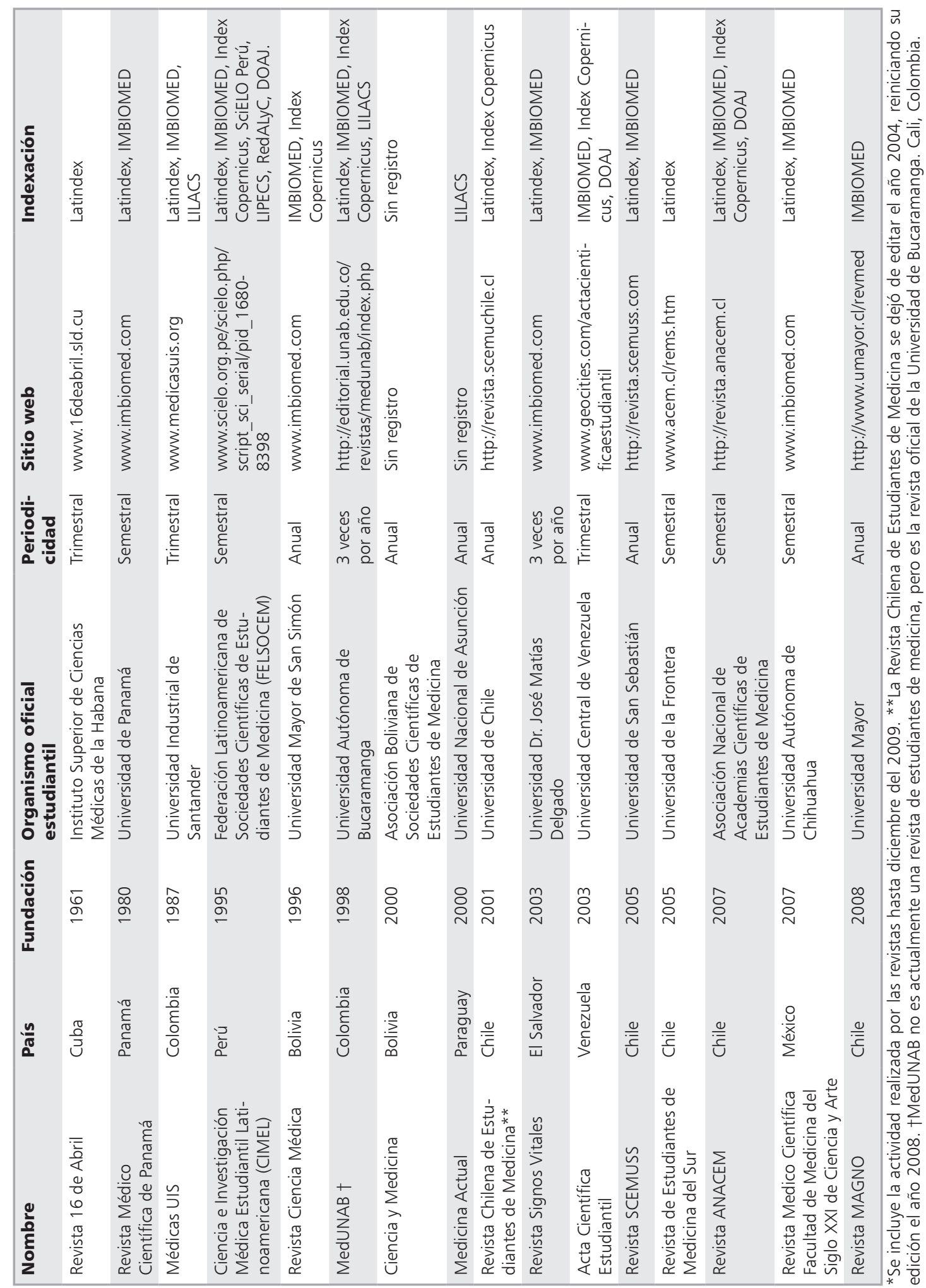


Revistas científicas de estudiantes de medicina - I. Cabrera-Samith et al

Investigación Médica Estudiantil Latinoamericana (CIMEL), indexada en SciELO. La mayoría de estas revistas están indexadas en distintas bases de datos biomédicas locales, regionales e internacionales. La Tabla 2 muestra una lista de estas revistas, detallando sus diferentes características, actualizadas hasta el año 2009.

\section{¿Qué viene próximamente para las revistas estudiantiles en Latinoamérica?}

Del mismo modo como se ha expandido la investigación en el pregrado entre los estudiantes de medicina de Latinoamérica, se espera que también se produzca un crecimiento en el número de revistas científicas de estudiantes de pregrado. Por lo tanto, se hace necesaria la creación de una asociación oficial, apoyada por FELSOCEM, que reúna a todos los editores de revistas científicas estudiantiles de Latinoamérica con el fin de mejorar la calidad científica, aumentar la tasa de indexación de todas las revistas y reunir sus esfuerzos para motivar a los estudiantes de medicina a publicar sus investigaciones. Además, también es importante la creación de un sistema de indexación exclusivo para revistas de estudiantes de pregrado, como SciELO, donde pueden tener un lugar que les dé la posibilidad de ser vistas no solamente por los propios estudiantes, de tal manera que los artículos publicados por estudiantes puedan ser citados por toda la comunidad médica en sus investigaciones. Actualmente, sólo existe una iniciativa con esta idea, desde el año 2009, en la base de datos IMBIOMED (www.imbiomed.com.mx) donde existe una sección especial que agrupa a las principales revistas científicas cuyo comité editorial está compuesto por estudiantes de pregrado.

\section{Referencias}

1. Franco C, Rodríguez-Morales AJ. Revistas científicas de estudiantes de medicina. Gac Med Caracas 2009; 11: 70, 90.

2. Huamaní C, Mayta-Tristán P, Rodríguez-Morales AJ. Publicar desde pregrado. Interciencia 2008 33: 785.

3. Steven L. Student journals: Facilitating medical student research and physician-scientist development. McGill J Med 2006; 9: 82-83.

4. Aslam F, Shakir M, Qayyum MA. Why medical students are crucial to the future of research in South Asia. PLoS Medicine 2005; 2: e322 doi:10.1371/journal. pmed.0020322.

5. Marusic A, Marusic M. Teaching students how to read and write science: a mandatory course on scientific and communication in medicine. Acad Med 2003; 78: 1235-9.

6. Gutiérrez C, Mayta-Tristán P. Publicación desde el pregrado en Latinoamérica: importancia, limitaciones y alternativas de solución. CIMEL 2003 8: 53-60.

7. Molina-Ordoñez J, Huamaní C, Mayta-Tristán P. Apreciación estudiantil sobre la capacitación universitaria en investigación: estudio preliminar. Rev Peru Med Exp Salud Pública 2008; 25: 325-9.

8. Mayta-Tristán P. Revistas científicas estudiantiles en Latinoamérica. Rev Med Chile 2006; 134: 395-7.

9. Ángulo-Bazán Y. Situación actual de las revistas científicas estudiantiles en Latinoamérica. CIMEL 2009; 13: 36-7.

10. Orostegui-Pinilla D, Cabrera-Samith I, Ángulo-Bazán Y, Mayta-Tristán P, Rodríguez-Morales AJ. Encuentros internacionales de revistas científicas estudiantiles de las ciencias de la salud en Latinoamérica, 1998-2008. Rev Panam Salud Pública 2009; 25: 469-70.

11. Cabrera-Samith I, Garrido F. El desarrollo de las revistas científicas de estudiantes de medicina en Chile. Rev Med Chile 2009; 137: 1265-6. 